\title{
Trade-off Analysis of Energy-Efficiency versus Generated Interference when Using Regenerative Relay
}

\author{
Goran Dimić, Member, IEEE, Dragana Bajić, Member, IEEE, and Marko Beko, Member, IEEE
}

\begin{abstract}
When using a regenerative relay, a trade-off between increasing the energy efficiency versus reducing the generated interference arises. In this paper, the generated interference in the direct link from the base station to a user terminal is compared with the generated interference from both the base station and the relay node in regenerative relay transmissions. In the space of channel losses, in the transceivers' operating region, the lines corresponding to the generated interference ratio comparing the relay scheme and the direct link are depicted. It is shown that it is possible to tune the energy-efficiency vs generated interference trade-off, by adjusting the transmit powers, constellation size and code rate. The trade-off depends on the channel losses along the links. A key enabler of the trade-off is the appropriate placement of the relay node.
\end{abstract}

Keywords - energy efficiency, hetnets, interference reduction, power control, regenerative relay, wireless.

\section{INTRODUCTION}

$\mathrm{T}$ HE use of a regenerative relay (RR) may improve several performance metrics in wireless communications [1], and in the LTE-Advanced in particular [2]. A holistic approach to energy efficiency (EE) in mobile radio networks is presented in [3]. We consider improving the EE by using RR, where the metric is the number of bits transmitted per unit of energy [bits/J].

The work [4] focused on EE during the downlink data transmission in the cellular scenario. It shows the EE ratio between the RR and the direct link in the space of channel

Paper received April 20, 2015; revised Jun 22, 2015; accepted Jun 27, 2015. Date of publication July 15, 2015. The associate editor coordinating the review of this manuscript and approving it for publication was Prof. Aleksandar Nešković.

This paper is a revised and expanded version of the paper presented at the 22th Telecommunications Forum TELFOR 2014.

This paper is supported in part by grants TR32043 and III43002 of the Ministry of Education and Science of the Republic of Serbia, grant APV 114-451-2061/2011-01, bilateral cooperation project Portugal-Serbia grant 451-03-02338/2012-14/11, and PTDC/EEI-TEL/2990/2012-ADIN and EXPL/EEITEL/0969/2013 grants by Fundação para a Ciência e a Tecnologia of Portugal.

Goran Dimić, University of Belgrade, Institute Mihailo Pupin, Volgina 15, 11060 Belgrade, Serbia (e-mail: goran.dimic@pupin.rs) Dragana Bajić, University of Novi Sad, Faculty of Technical Sciences, Trg Dositeja Obradovića 6, 21000 Novi Sad, Serbia (e-mail: dragana.bajic@gmail.com)

Marko Beko, Universidade Lusofona de Humanidades e Technologias, Lisbon and UNINOVA-Campus FCT/UNL, Caparica, Portugal (e-mail: beko.marko@gmail.com). M. Beko is a collaborative member of INESC-INOV, Instituto Superior Tecnico, University of Lisbon, Lisbon, Portugal. losses of the former and both relay hops. The EE ratio can be greater than one, by increasing the RR data rate as much as possible. This increases the spectral efficiency (SE) of the relay scheme as well, to (partially) compensate for the loss of SE due to doubling of the number of time slots when using RR and possibly due to using 2 channels.

However, this requires increased transmit power, which in turn increases generated interference. Reducing the generated interference is beneficial, because a large number of non-distingushable interference components can limit the capacity of a wireless system, even if strong interference components are cancelled-out [5]. A careful design is required to achive desired performance metrics, while limiting the interference, when using the RR [6].

This paper analyzes interference at an unintended receiver $\mathrm{A}$, during the downlink transmission, in cases of the direct link and the two-hop RR, generated by the base station (BS) and the relay node (RN). In particular, the paper analyzes the variations of interference channel losses at the unintended receiver. Then, it combines the EE analysis of the RR, from previous work, with the analysis of the generated interference to formulate their trade-off. It is shown that the trade-off depends on the channel losses along the direct link, both RR links and the interference links. The benefits of the trade-off are enabled by a good BS-RN link, achieved by appropriate RN placement.

The rest of the paper is structured as follows. Section II presents the scenario, the energy consumption model of the RR and the direct link, and the channel loss model. Sec. III considers power consumption characteristics of the $\mathrm{BS}$ and the $\mathrm{RN}$, and derives the generated interference ratios. Sec. IV provides an assessment of a key parameter which influences the generated interference ratios. Sec. V provides an example. Sec. VI discusses the results of the assessment. Sec. VII concludes the paper.

\section{SCENARIO, ENERGY CONSUMPTION MODEL AND CHANNEL LOSS MODEL}

\section{A. Scenario}

Fig. 1 shows a two-hop $\mathrm{RR}$ in the cellular scenario downlink transmission, where the BS is the source (S), a user terminal (UE) is the destination (D), and the RN is a relay $(\mathrm{R})$. The direct link BS-UE is shown as a dashed line with arrow denoting downlink. The two-hop relay, BS-RN and RN-UE, is depicted by two solid lines with arrows denoting downlink. The figure also shows an unintended receiver, A, which is actually a UE in other cell. The 
signals it receives from the BS during either the direct link or the first hop of the RR, and the signals that A receives from the RN during the second hop of the RR are interference. The interference links are represented by dash-dotted lines. Throughout the paper, we use notation shown in Fig. 1. The subscripts $i=$ void, 1, 2, denote the direct link (BS-UE), the first relay link (BS-RN) and the second relay link (RN-UE), respectively. For each RR link, we use term hop. The subscripts A and A2 denote the interference links BS-A and RN-A, respectively.

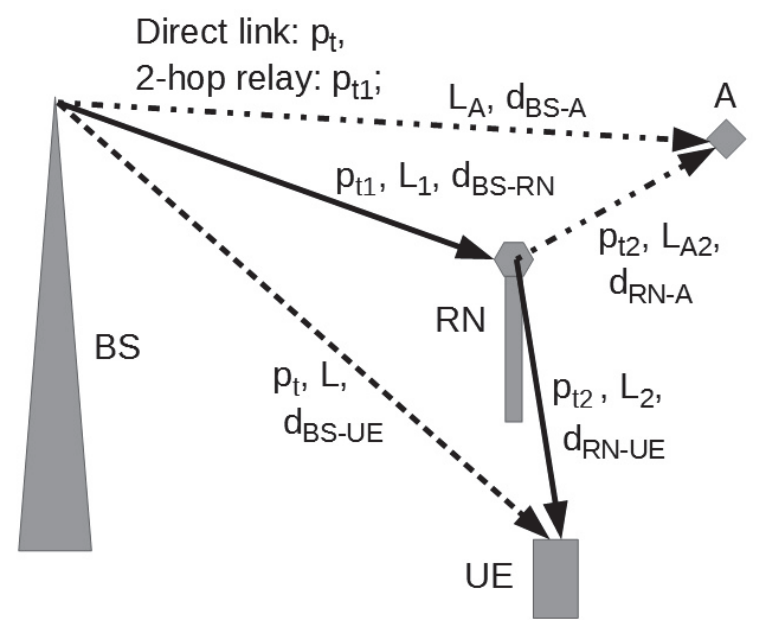

Fig. 1. Scenario of the downlink transmission: the direct link (BS-UE) and the 2-hop RR (BS-RN and RN-UE). There is an unintended receiver $\mathrm{A}$.

$p_{t i}$ denotes the transmit power at the transmitter (TX) of the link $i$. $p_{T X i}$ denotes the total TX power consumption of the link $i$ (not shown in Fig. 1).

$L_{i}$ denotes the channel loss in $\mathrm{dB}$ over the link $i$.

$d$ denotes the separating distance between a transmitterreceiver $(\mathrm{RX})$ pair, where subscript denotes TX-RX pair.

$p_{r i}$ and $p_{R X i}$ denote the received signal power and the total RX power consumption, respectively, at the RX of the link $i$. $p_{R X i}$ is assumed constant for each RX $i$. Values of $p_{t i}, p_{T X i}, p_{r i}$ and $p_{R X i}$ are obtained from [2], [7].

$P_{t h}$ denotes the $p_{r}$ threshold for successful reception, which depends on several parameters [8].

$g_{t}$ and $g_{r}$ denote the antenna gains at the TX and RX, respectively.

$r$ and $r_{R}$ denote the data rate achievable over the direct link and RR, respectively.

We use the following assumptions:

- A deterministic condition for successful reception, $p_{r} \geq P_{t h}$.

- The perfect power control to adjust $p_{t}$ at all transmitters (BS and $\mathrm{RN}$ ) to yield $p_{r}=P_{t h}$ at all the receivers.

- Both relay hops (BS-RN and RN-UE) have the same data rate, $2 r_{R}$, i.e., $P_{t h, 1}=P_{t h, 2}$.

\section{B. Energy Consumption and Energy Efficiency Ratios}

We use the total TX power consumption model, $p_{T X}=$ $f\left(p_{t}\right)$, of [8], with constraints $p_{t} \in\left[p_{t, \text { min }}, p_{t, \max }\right]$ and $p_{T X} \epsilon$ $\left[p_{T X, \min }, p_{T X, \max }\right]$.

An EE comparison between the RR and the direct link, is based on a simplified link budget

$$
p_{r}=g_{t} g_{r} p_{t} / L
$$

which yields [9]

$$
\begin{gathered}
L_{\max }=g_{t} g_{r} p_{t, \text { max }} / P_{t h}, \quad L_{\text {min }}=g_{t} g_{r} p_{t, \text { min }} / P_{t h}, \\
p_{t}=\left(P_{t h} L\right) /\left(g_{t} g_{r}\right) .
\end{gathered}
$$

Comparing the two-hop and single-hop transmissions, the energy consumption ratio $\alpha$ [8], the data rate ratio, $\beta$, and the EE ratio, $\eta$, [4], are:

$$
\begin{aligned}
& \alpha=\frac{\left(E_{T X, 1}+E_{R X, 1}\right)+\left(E_{T X, 2}+E_{R X, 2}\right)}{\left(E_{T X}+E_{R X}\right)}, \\
& \beta\left(p_{t}, p_{t 1}, p_{t 2}\right)=\frac{r_{R}\left(p_{t 1}, p_{t 2}\right)}{r\left(p_{t}\right)}, \\
& \eta\left(p_{t}, p_{t 1}, p_{t 2}\right)=\frac{\beta\left(p_{t}, p_{t 1}, p_{t 2}\right)}{\alpha\left(p_{t}, p_{t 1}, p_{t 2}\right)} .
\end{aligned}
$$

Here, $\alpha$ is evaluated for the same $\left(p_{t}, p_{t 1}, p_{t 2}\right)$ as $\beta$ and $\eta$. Increased $\beta$ increases $\eta$ [4]. Under a typical downlink scenario, $\alpha$ increases at a smaller rate than $\beta$.

It is shown in [4] that it is possible to increase $r_{R}$ w.r.t. $r$ $(\beta \geq 1)$, by increasing the constellation and adjusting the code rate along both relay hops. For instance, for QAM constellations used in OFDM, approximately, if $P_{t h, 1} \geq P_{t h}$ $+6 \mathrm{~dB}$, then $\beta \geq 1$ [4]. If $\beta=1$, then both the direct link and the RR transmit the same amount of data in two slots: the former half of data in each slot, the latter all data in each hop. We use the assumption $\beta=1$ in the rest of the paper.

\section{Channel Loss Model}

The trade-off between improving EE and limiting the generated interference using RR, is based on exploiting the differences between channel losses along the direct link and relay hops. To demonstrate this claim, we use a modified Urban channel loss model for relay communications [9], which accounts for the path loss and shadowing, in both line-of-sight (LOS) and non-LOS (NLOS) channel types (log stands for $\log _{10}$ ):

$$
L_{i}[\mathrm{~dB}]=\left\{\begin{array}{c}
{\left[b_{i}+10 n_{i} \log d_{i}\right]+} \\
+m_{i} \sigma_{i}\left[1-\exp \left(-\frac{d_{i}-d_{0}}{D_{S, i}}\right)\right], \\
{\left[-27.6+20 \log d_{i}\right]+} \\
+m_{i} 2\left[1-\exp \left(-\frac{d_{i}}{30}\right)\right], \text { LOS }
\end{array}\right.
$$

where subscript $i$ denotes the link. The left square brackets in the sum model the path loss and the right additive term models shadowing, with standard deviation $\sigma_{i} . d_{i}$ denotes the TX-RX distance of the link $i$, and is expressed in meters. The parameters are: $b_{i}$, expressed in $\mathrm{dB}, n_{i}$, a dimensionless number, and $d_{0}$ and $D_{S, i}$, expressed in meters. Their values are provided in the model [9] (Table I and II). The LOS case has the same channel loss function (bottom case in (5)) irrespective of the TX-RX pairs [9]. Parameter $m_{i}$ is the multiplicative factor of the shadowing standard deviation. It is introduced in this paper to serve as a shadowing margin, which may depend on the shadowing distribution or receiver design requirements. In NLOS case, the shadowing distribution is confirmed to be lognormal, whereas in LOS case it is more similar to a Uniform distribution [9]. Fig. 2 shows the NLOS channel losses of the links, individually, with $m_{i}=1, m_{i}=3$, and the LOS curve being equal for all three TX-RX pairs, but 
two distinct shadowing variations $(2 \mathrm{~dB}, 6 \mathrm{~dB})$.

The conditional probability of LOS as a function of the separating distance between the transmitter and receiver is given in [9 (Fig. 4)]. This probability is 1 for the separating distance less than 10, and non-negligible for the separating distance of less than 200 [meters]. The conditional LOS probability of the BS-RN link dominates the BS-UE link, and the BS-UE link dominates the RN-UE link over all separating distances.

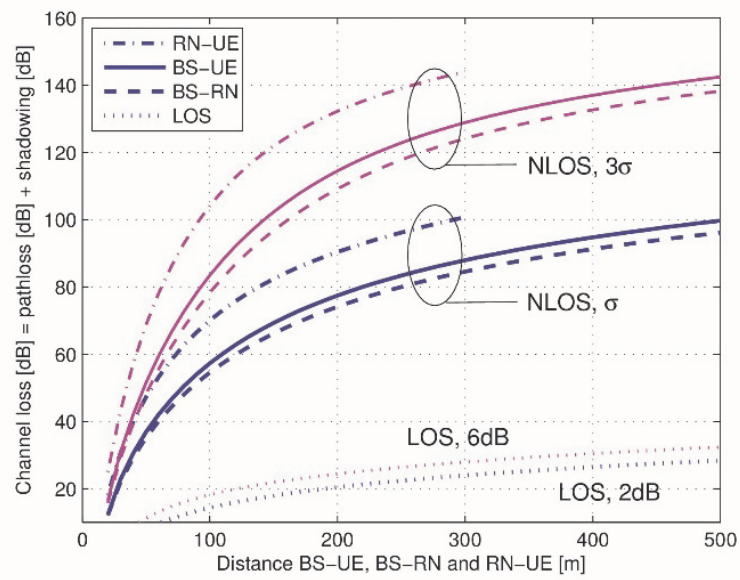

Fig. 2. Channel losses along the links: BS-UE, BS-RN and RN-UE.

\section{GENERATED INTERFERENCE LEVELS IN THE SPACE OF CHANNEL LOSSES}

The interference power the BS makes at an unintended receiver - a point A, during both slots of the direct link, is

$$
I_{A}=p_{r \mathrm{~A}}^{B S-U E}=\frac{p_{t} g_{S} g_{D}}{L_{\mathrm{A}}}=P_{t h} \frac{L}{L_{\mathrm{A}}},
$$

where $p_{t}$ is determined from (3).

The interference power of the BS in slot 1, and the RN in slot 2, at the point A, when RR is used, are

$$
\begin{gathered}
I_{\mathrm{A}}^{R 1}=p_{r \mathrm{~A}}^{B S-R N}=\frac{p_{t 1} g_{S} g_{D}}{L_{\mathrm{A}}}=P_{t h, 1} \frac{g_{D}}{g_{R}} \frac{L_{1}}{L_{\mathrm{A}}}, \\
I_{\mathrm{A}}^{R 2}=p_{r \mathrm{~A}}^{R N-U E}=\frac{p_{t 2} g_{R} g_{D}}{L_{\mathrm{A} 2}}=P_{t h, 1} \frac{L_{2}}{L_{\mathrm{A} 2}},
\end{gathered}
$$

where $p_{t 1}$ and $p_{t 2}$ are determined from (3) for $L_{1}$ and $L_{2}$, respectively. Parameters $g_{D}$ and $g_{R}$ are given and constant; $P_{t h}$ and $P_{t h, 1}$ are given, but may be adjusted. $L, L_{1}, L_{2}$ are obtained from the model (5) for given positions of the BS, RN and UE (Fig. 2).

Point A being a UE narrows the results of our paper, but keeps the analysis within the Urban channel model (5). If A were another BS or RN, we would need the channel models BS-BS, RN-BS and BS-RN, which are not provided in [9]. Hence, we consider: $b_{\mathrm{A}}=b, b_{\mathrm{A} 2}=b_{2}, n_{\mathrm{A}}=n$, $n_{\mathrm{A} 2}=n_{2}, \sigma_{\mathrm{A}}=\sigma, \sigma_{\mathrm{A} 2}=\sigma_{2}, D_{S, \mathrm{~A}}=D_{S}, D_{S, \mathrm{~A} 2}=D_{S, 2}$.

Variables $L_{\mathrm{A}}$ and $L_{\mathrm{A} 2}$ are unknown, but have the same path loss and shadowing model as $L$ and $L_{2}$, respectively, c.f. (5). Multipliers $m_{\mathrm{A}}$ and $m_{\mathrm{A} 2}$ may not be equal to $m$ and $m_{2}$, respectively. There may be correlation between $L_{\mathrm{A}}$ and $L$, and/or between $L_{\mathrm{A} 2}$ and $L_{2}$. Consideration of the correlations is beyond the scope of this paper, but by alowing the difference in multipliers we enable the case of uncorrelated channel losses. Let the interference channel losses ratio at a point $\mathrm{A}$, denoted $\tau$, be

$$
\tau=\frac{L_{\mathrm{A} 2}}{L_{\mathrm{A}}} .
$$

In logarithmic scale, $x[\mathrm{~dB}]=x^{\mathrm{dB}}, \tau^{\mathrm{dB}}=L_{\mathrm{A} 2}{ }^{\mathrm{dB}}-L_{\mathrm{A}}{ }^{\mathrm{dB}}$. Let

$$
\kappa_{1}=\frac{I_{\mathrm{A}}^{R 1}}{I_{\mathrm{A}}}, \quad \kappa_{2}=\frac{I_{\mathrm{A}}^{R 2}}{I_{\mathrm{A}}}
$$

for a feasible ratio $\tau$. In logarithmic scale, the relationships in the space of channel losses $\left(L, L_{1}, L_{2}\right)$, are

$$
\begin{gathered}
L_{1}^{\mathrm{dB}}=L^{\mathrm{dB}}+\Delta P_{t h}^{\mathrm{dB}}+\Delta g^{\mathrm{dB}}+\kappa_{1}^{\mathrm{dB}}, \\
L_{2}^{\mathrm{dB}}=L^{\mathrm{dB}}+\Delta P_{t h}^{\mathrm{dB}}+\tau^{\mathrm{dB}}+\kappa_{2}^{\mathrm{dB}} .
\end{gathered}
$$

Note that $\kappa_{i}^{\mathrm{dB}}<0 \mathrm{~dB}\left(\kappa_{i}<1\right)$ means that interference is reduced when using the relay link $i$ w.r.t. the direct link in hop $i$, for $i=1,2$. Ideally, the values of variables and parameters in (11) and (12) will enable $\kappa_{i}^{\mathrm{dB}}<0 \mathrm{~dB}$, but this is not guaranteed.

In (11), the key to lower $\kappa_{1}$ is decrease of $L_{1}$ w.r.t. $L$ by appropriate placement of the $\mathrm{RN}$ w.r.t. the BS, e.g. by providing LOS. The increase of $\Delta g$ enables decrease of $\kappa_{1}$, but the change is limited to a few $\mathrm{dB}$.

In (12), a key to lower $\kappa_{2}$ is decrease of $L_{2}$ w.r.t. $L$, but this depends on the terrain and buildings along the RN-UE link, which are not under network designer's control. Another key is attaining a desired value of $\tau$, which is also not under network designer's control. However, the region of points $\mathrm{A}$ in which desirable values of $\tau$ are achievable can be determined.

\section{ANALYSIS OF INTERFERENCE CHANNEL LOSSES RATio AT A POINT A}

Because of the influence of $\tau$ on $\kappa_{2}$ (12), we are interested in the range of values of $\tau$ for various possible values of path-loss and shadowing parameters (c.f. (5)), and separating distances BS-A and RN-A. We partition the cases based on the LOS/NLOS conditions, in Table 1.

The analytical expression for evaluation of $\tau$ for the four cases $c 1-c 4$ is (log stands for $\left.\log _{10}\right)$ is:

$$
\tau^{\mathrm{dB}}=\left\{\begin{array}{c}
20 \log d_{\mathrm{A} 2}+2 m_{\mathrm{A} 2}\left[1-\exp \left(-\frac{d_{\mathrm{A} 2}}{30}\right)\right]- \\
-20 \log d_{\mathrm{A}}-2 m_{\mathrm{A}}\left[1-\exp \left(-\frac{d_{\mathrm{A}}}{30}\right)\right], \\
b_{2}+10 n_{2} \log d_{\mathrm{A} 2}+m_{\mathrm{A} 2} \sigma_{2}\left[1-\exp \left(-\frac{d_{\mathrm{A} 2}-d_{0}}{D_{S, 2}}\right)\right]+ \\
+27.6-20 \log d_{\mathrm{A}}-2 m_{\mathrm{A}}\left[1-\exp \left(-\frac{d_{\mathrm{A}}}{30}\right)\right], \\
-27.6+20 \log d_{\mathrm{A} 2}+2 m_{\mathrm{A} 2}\left[1-\exp \left(-\frac{d_{\mathrm{A} 2}}{30}\right)\right]- \\
-b-10 n \log d_{\mathrm{A}}+m_{\mathrm{A}} \sigma\left[1-\exp \left(-\frac{d_{\mathrm{A}}-d_{0}}{D_{S}}\right)\right], \\
b_{2}+10 n_{2} \log d_{\mathrm{A} 2}+m_{\mathrm{A} 2} \sigma_{2}\left[1-\exp \left(-\frac{d_{\mathrm{A} 2}-d_{0}}{D_{S, 2}}\right)\right]- \\
c 3 \\
-b-10 n \log d_{\mathrm{A}}-m_{\mathrm{A}} \sigma\left[1-\exp \left(-\frac{d_{\mathrm{A}}-d_{0}}{D_{S}}\right)\right], \\
c 4
\end{array}\right.
$$

A reference value of $\tau$ corresponds to $\kappa_{2}{ }^{\mathrm{dB}}=0 \mathrm{~dB}$ :

$$
\tau_{0}^{\mathrm{dB}}=L_{2}^{\mathrm{dB}}-L^{\mathrm{dB}}-\Delta P_{t h}^{\mathrm{dB}} .
$$

Note that $\tau$ may not be correlated to $L_{2}$ and $L$, so that $\tau^{\mathrm{dB}}$ 
may deviate significantly from $\tau_{0}^{\mathrm{dB}}$. If $\tau^{\mathrm{dB}}>\tau_{0}{ }^{\mathrm{dB}}$ then $\kappa_{2}{ }^{\mathrm{dB}}<0 \mathrm{~dB}$, which favors the use of the RR in the sense that the generated interference is reduced. The opposite favors the direct link BS-UE.

TABLE 1: POSSIBLE LOS/NLOS CONDITIONS OVER RN-A AND BS-A LINKS.

\begin{tabular}{|c|c|c|c|c|}
\hline & C1 & C2 & C3 & C4 \\
\hline RN-A & LOS & NLOS & LOS & NLOS \\
\hline BS-A & LOS & LOS & NLOS & NLOS \\
\hline
\end{tabular}

The evaluation of $\tau$ requires known distances BS-A and $\mathrm{RN}-\mathrm{A}$, as well as known LOS/NLOS conditions. Alternatively, one can evaluate $d_{\mathrm{A} 2}$ for the given LOS/NLOS conditions, $d_{\mathrm{A}}$ and desired $\tau^{\mathrm{dB}}$. This defines the region in which the interference from the $\mathrm{RN}$ during the second hop of RR is below the interference from the BS during the second slot.

There is a wide interval of values of $\tau$. For instance, for distances greater than $20 \mathrm{~m}$ from the $\mathrm{RN}, \tau$ ranges from less than $-50 \mathrm{~dB}$ to more than $50 \mathrm{~dB}$. The dependence of $\tau$ on the independent BS-A and RN-A distances, in NLOS/NLOS case, is shown in Fig. 3. Equipotential lines of $\tau$ having values of $[-50,-20,0,20,50] \mathrm{dB}$ appear in ascending order going counter-clockwise from the $\mathrm{x}$-axis. The solid lines stretching from the lower left corner are $\tau$ levels. The dashed line and dash-dotted line show the distance between the $\mathrm{BS}$ and $\mathrm{RN}$ of $400 \mathrm{~m}$ and $300 \mathrm{~m}$, respectively. For a given BS-RN distance, the feasible values of $\tau$ lie to the right and above the BS-RN distance line, because of the triangle inequality $d_{\mathrm{A}}+d_{\mathrm{A} 2} \geq d_{\mathrm{BS}-\mathrm{RN}}$.

\section{A. Variations in $\tau$ Ratio}

The dependence of $\tau$ on the variation of channel losses due to change in shadowing deviation/margin along both BS-A and RN-A links can be assessed from

$$
\begin{array}{cl}
\frac{\partial \tau^{\mathrm{dB}}}{\partial m_{\mathrm{A}}}= \begin{cases}-2\left[1-\exp \left(-\frac{d_{\mathrm{A}}}{30}\right)\right], & c 1, c 2 \\
-\sigma\left[1-\exp \left(-\frac{d_{\mathrm{A}}-d_{0}}{D_{S}}\right)\right], & c 3, c 4\end{cases} \\
\frac{\partial \tau^{\mathrm{dB}}}{\partial m_{\mathrm{A} 2}}= \begin{cases}2\left[1-\exp \left(-\frac{d_{\mathrm{A} 2}}{30}\right)\right], & c 1, c 3 \\
\sigma_{2}\left[1-\exp \left(-\frac{d_{\mathrm{A} 2}-d_{0}}{D_{S, 2}}\right)\right], & c 2, c 4\end{cases}
\end{array}
$$

It follows that

$$
\frac{\partial \tau^{\mathrm{dB}}}{\partial m_{\mathrm{A}}}<0 \text { and } \quad \frac{\partial \tau^{\mathrm{dB}}}{\partial m_{\mathrm{A} 2}}>0
$$

in all cases, for all separating distances and shadowing deviations. In the case of interest when point $\mathrm{A}$ is outside the cell of interest, closer to the RN than the $\mathrm{BS}, d_{\mathrm{A}}>d_{\mathrm{A} 2}$, $\tau^{\mathrm{dB}}$ is somewhat more sensitive to changes in shadowing deviation/margin along the $\mathrm{RN}-\mathrm{A}$ link than along the BSA link. It is shown in Fig. 4. For instance, when the RN-A channel improves relative to the BS-A channel for the given point $\mathrm{A}$ (case $\left.\tau\left(3 \sigma, \sigma_{2}\right)\right)$, $\tau$ decreases. While the bending of the $\tau=0 \mathrm{~dB}$ line is significant, as the channel losses change, the bending of the $\tau=-50 \mathrm{~dB}$ and $\tau=50 \mathrm{~dB}$ is less pronounced.

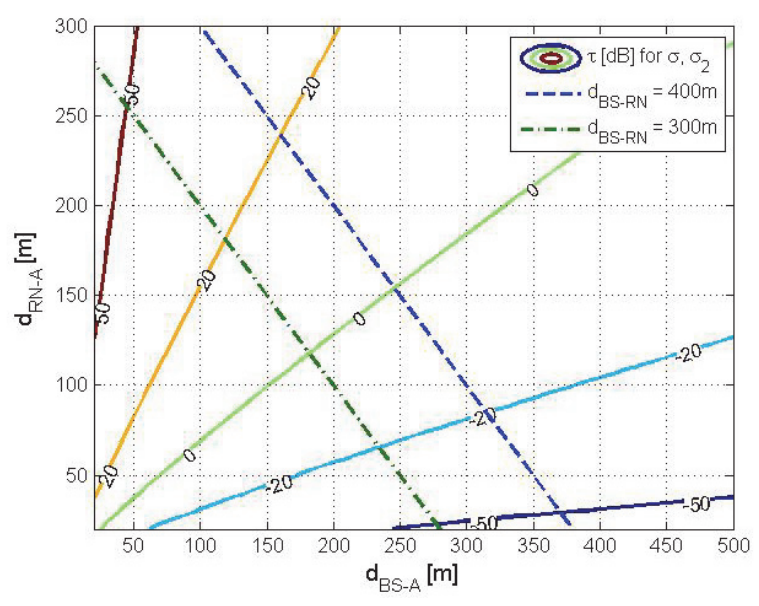

Fig. 3. $\tau[\mathrm{dB}]$ depending on distances BS-A (x-axis) and RN-A (y-axis).

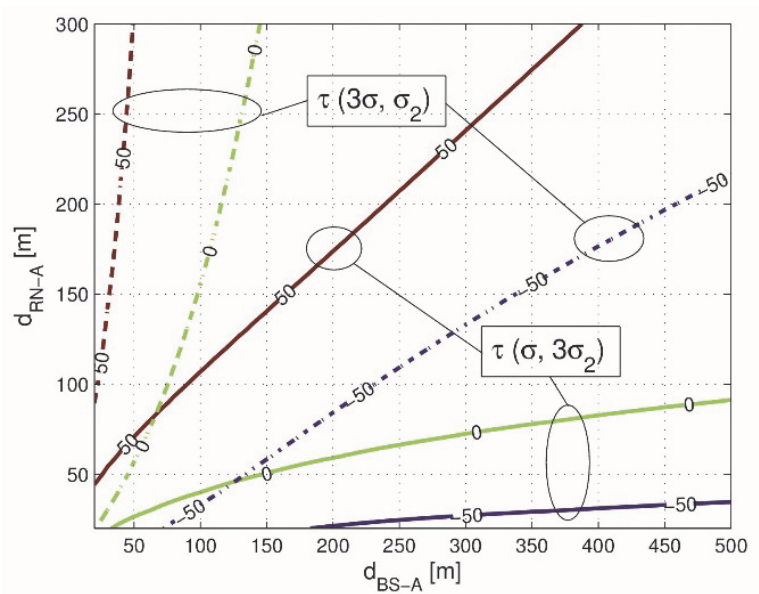

Fig. 4. $\tau[\mathrm{dB}]$ depending on distances BS-A (x-axis) and RN-A (y-axis), NLOS/NLOS.

A different perspective on changes of $\tau$ is obtained when taking into account change in separating distances BS-A and $\mathrm{RN}-\mathrm{A}$.

$$
\begin{gathered}
\frac{\partial \tau^{\mathrm{dB}}}{\partial d_{\mathrm{A}}}=\left\{\begin{array}{ll}
-\frac{20}{d_{\mathrm{A}} \ln 10}-\frac{m_{\mathrm{A}}}{15} \exp \left(-\frac{d_{\mathrm{A}}}{30}\right), & c 1, c 2 \\
-\frac{10 n}{d_{\mathrm{A}} \ln 10}-\frac{m_{\mathrm{A}} \sigma}{D_{S}} \exp \left(-\frac{d_{\mathrm{A}}-d_{0}}{D_{S}}\right), & c 3, c 4
\end{array},\right. \\
\frac{\partial \tau^{\mathrm{dB}}}{\partial d_{\mathrm{A} 2}}=\left\{\begin{array}{ll}
-\frac{20}{d_{\mathrm{A} 2} \ln 10}-\frac{m_{\mathrm{A} 2}}{15} \exp \left(-\frac{d_{\mathrm{A}}}{30}\right), & c 1, c 3 \\
-\frac{10 n_{2}}{d_{\mathrm{A} 2} \ln 10}-\frac{m_{\mathrm{A} 2} \sigma_{2}}{D_{S, 2}} \exp \left(-\frac{d_{\mathrm{A} 2}-d_{0}}{D_{S, 2}}\right), & c 2, c 4
\end{array} .\right.
\end{gathered}
$$

It follows that

$$
\frac{\partial \tau^{\mathrm{dB}}}{\partial d_{\mathrm{A}}}<0, \text { and } \frac{\partial \tau^{\mathrm{dB}}}{\partial d_{\mathrm{A} 2}}>0
$$

in all cases, for all separating distances, shadowing deviations and margins. For distances $d_{\mathrm{A}}=400 \mathrm{~m}$ and $d_{\mathrm{A} 2}=$ $100 \mathrm{~m}, \tau^{\mathrm{dB}}$ is around 4 times more sensitive to the change of $d_{\mathrm{A} 2}$ than to the change of $d_{\mathrm{A}}$. An example is given in Fig. 5, which shows the values of $\tau^{\mathrm{dB}}$ around the $\mathrm{RN}$, assuming the Urban model of channel losses (5). The BS$\mathrm{RN}$ distance is $400 \mathrm{~m}$, in NLOS/NLOS case, with shadowing deviations $\left(\sigma, \sigma_{2}\right)$. The shown values change from $-80 \mathrm{~dB}$ to $-5 \mathrm{~dB}$ as the distance from the $\mathrm{RN}$ increases. In Fig. 5, at the distance of approximately $50 \mathrm{~m}$ from the 
$\mathrm{RN}, \tau^{\mathrm{dB}} \in(-40,-50) \mathrm{dB}$. It can be shown that for NLOS/NLOS case with shadowing deviations $\left(\sigma, 3 \sigma_{2}\right)$, at the distance $d_{\mathrm{A} 2} \approx 50$ meters, $\tau^{\mathrm{dB}} \in(-20,-30) \mathrm{dB}$.

The values of $\tau^{\mathrm{dB}}$ around the RN change little if the BS$\mathrm{RN}$ distance is changed between $300 \mathrm{~m}$ and $500 \mathrm{~m}$.

We note that $\tau^{\mathrm{dB}}$ typically changes more due to changes in shadowing deviation/margin than due to changes in separating distances. This is partly because the relative change of shadowing deviation/margin is larger than the relative change in the separating distances ratio, $d_{\mathrm{A} 2} / d_{\mathrm{A}}$.

\section{B. A Generalized Model of Changes in $\tau$ Ratio}

To assess all possible changes of $\tau$, including changes in LOS/NLOS conditions, we propose a simple generalized model of changes in channel losses. Let

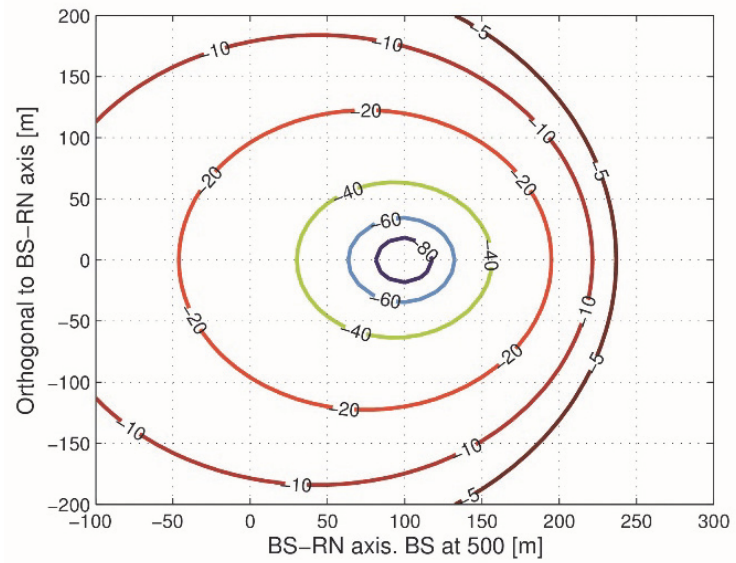

Fig. 5. Equipotential $\tau$ lines around the RN, against a 2-D "map": BS is at $(500,0)[\mathrm{m}], \mathrm{RN}$ is at $(100,0)[\mathrm{m}]$.

$$
\begin{aligned}
& L_{\mathrm{A}}^{\mathrm{dB}}=L_{\mathrm{A}}^{\overline{\mathrm{dB}}}+\Delta L_{\mathrm{A}}^{\mathrm{dB}}, \\
& L_{\mathrm{A} 2}^{\mathrm{dB}}=L_{\mathrm{A} 2}^{\overline{\mathrm{dB}}}+\Delta L_{\mathrm{A} 2}^{\mathrm{dB}} .
\end{aligned}
$$

Then

$$
\tau^{\mathrm{dB}}=\tau^{\overline{\mathrm{dB}}}+\Delta \tau^{\mathrm{dB}},
$$

where

$$
\begin{aligned}
& \tau^{\overline{\mathrm{dB}}}=L_{\mathrm{A} 2}^{\overline{\mathrm{dB}}}-L_{\mathrm{A}}^{\overline{\mathrm{dB}}}, \\
& \Delta \tau^{\mathrm{dB}}=\Delta L_{\mathrm{A} 2}^{\mathrm{dB}}-\Delta L_{\mathrm{A}}^{\mathrm{dB}} .
\end{aligned}
$$

For instance, one may set $L_{\mathrm{A}}^{\overline{\mathrm{d} B}}$ and $L_{\mathrm{A} 2}^{\overline{\mathrm{d}}}$ to correspond to NLOS case with multipliers $m_{\mathrm{A}}=1, m_{\mathrm{A} 2}=1$ and selected shadowing deviation. If the BS-A path has NLOS with stronger shadowing, then its multiplier, $m_{\mathrm{A}}$, is increased, so that $\Delta L_{\mathrm{A}}^{\mathrm{dB}}>0$. If the BS-A path has LOS, then its shadowing deviation is significantly reduced: $\Delta L_{\mathrm{A}} \mathrm{dB}^{\mathrm{B}}<0$ (c.f. (5)). The same holds for RN-A path and $\Delta L_{\mathrm{A} 2}{ }^{\mathrm{dB}}$.

As an illustration, the Table 2 shows discrete possibilities for $\Delta \tau^{\mathrm{dB}}$, when both RS-A and BS-A links experience NLOS (case $\mathrm{c} 4$ ), with two different shadowing margin values, relative to the $m_{\mathrm{A}}=1, m_{\mathrm{A} 2}=1$.

Table 3 expands Table 1 to show possibilities for $\Delta \tau^{\mathrm{dB}}$, depending on the existence of LOS, relative to case $\mathrm{c} 4$.

Existence of LOS along exactly one of the links (in cases $\mathrm{c} 2$ and $\mathrm{c} 3$ ), changes path loss parameters in addition to the shadowing margin/deviation relative to the NLOS link. Therefore, relative to the case $\mathrm{c} 4$ with changes in shadowing margin (see Table 2), cases c2 and c3 may have a larger absolute value of $\Delta \tau^{\mathrm{dB}}$.
TABLE 2: POSSIBLE NLOS/NLOS CONDITIONS OVER RN-A AND BS-A LINKS, WITH VARIATIONS IN MARGIN MULTIPLIERS.

\begin{tabular}{|c|c|c|c|c|}
\hline RN-A & $m_{\mathrm{A} 2}=1$ & $m_{\mathrm{A} 2}=3$ & $m_{\mathrm{A} 2}=1$ & $m_{\mathrm{A} 2}=3$ \\
\hline BS-A & $m_{\mathrm{A}}=1$ & $m_{\mathrm{A}}=1$ & $m_{\mathrm{A}}=3$ & $m_{\mathrm{A}}=3$ \\
\hline & $/$ & $\Delta \tau^{\mathrm{dB}}>0$ & $\Delta \tau^{\mathrm{dB}}<0$ & ANY \\
\hline
\end{tabular}

TABLE 3: Change IN TAU DUE TO VARIOUS LOS/NLOS CONDITIONS OVER RN-A AND BS-A LINKS.

\begin{tabular}{|c|c|c|c|c|}
\hline & C1 & C2 & C3 & C4 \\
\hline RN-A & LOS & NLOS & LOS & NLOS \\
\hline BS-A & LOS & LOS & NLOS & NLOS \\
\hline$\Delta \tau^{\mathrm{dB}}$ & ANY & $\Delta \tau^{\mathrm{dB}}>0$ & $\Delta \tau^{\mathrm{dB}}<0$ & $/$ \\
\hline
\end{tabular}

\section{AN EXAMPLE}

Assume an urban environment with a Micro BS, and Pico-BS-like characteristics of the RN. The total power consumption values are based on [7] for 2012 State-of-theArt. The values of $p_{t, \min }$ and $p_{t, \max }$ of the $\mathrm{BS}$ and $\mathrm{RN}$ are based on 3GPP requirements [10]. The minimum power is evaluated using the peak maximum power and the minimum requirements for Total power dynamic range (e.g. 16.9dB for $10 \mathrm{MHz}$ bandwidth [10]) [4]. An example is shown in Fig. 6, where the $\mathrm{x}$-axis is logarithmic $[\mathrm{dBm}]$, and the $\mathrm{y}$-axis is linear [W]. $\beta=1$, so that 2 slots are consumed in direct link (dash-dotted vertical lines with arrow on top: SLOT 1, SLOT 2). The difference in the range of $p_{T X}$, motivates the use of relay to improve the EE.

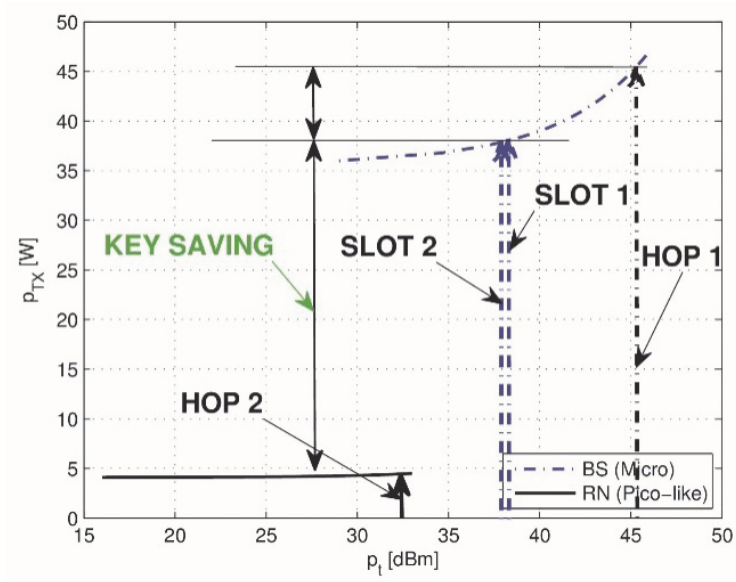

Fig. 6. Power consumption: Micro-BS and Pico-BS-like RN.

The antenna gains at the BS, RN and UE, respectively, are $g_{S}=15 \mathrm{~dB}, g_{R}=5 \mathrm{dBi}$ and $g_{D}=0 \mathrm{dBi}$.

We consider point $\mathrm{A}$ to be out of the cell of interest, and assume $\tau^{\mathrm{dB}} \approx-3 \mathrm{~dB}$ at the point $\mathrm{A}$ and around it. This value of $\tau^{\mathrm{dB}}$ appears at some distance from the cell edge, so that with high probability, both BS-A and RN-A links experience NLOS conditions. Larger values of $\tau^{\mathrm{dB}}$ are possible at larger distances, which favors the RR.

Assume equal BS-UE and BS-RN distances of $500 \mathrm{~m}$, so that we assume NLOS along both links (which occur with high probability), $L=142.4 \mathrm{~dB}$ and $L_{1}=138.2 \mathrm{~dB}$. The RNUE distance is $100 \mathrm{~m}$ with assumed NLOS condition, so that $L_{2}=104.2 \mathrm{~dB}$ (c.f. (1), see Fig. 2). Then, the operating point (OP) in the space of channel losses is $\left(L, L_{1}\right.$, $\left.L_{2}\right)=(142.4,138.2,104.2)$.

Fig. 7 shows achievable $\kappa_{1}, \kappa_{2}$ for $\tau=-3 \mathrm{~dB}$ at the OP. 
The axes limits correspond to $\left[L_{1, \min }, L_{1, \max }\right]$ and $\left[L_{2, \min }\right.$, $\left.L_{2, \max }\right]$, which are obtained from (2) for $P_{t h}=-85 \mathrm{dBm}$. The dotted lines with markers show $\alpha$-levels (4). The data rate ratio is leveled, $\beta=1$, due to selection of appropriate $P_{t h, 1}$, constellation and code rate over relay hops. At the OP, $\kappa_{1} \approx-3 \mathrm{~dB}, \kappa_{2} \approx-29 \mathrm{~dB}$, whereas the EE ratio, $\eta \approx 1.85$.

Note that by changing the OP, it is possible to increase $\eta$ at the expense of increased $\kappa_{1}, \kappa_{2}$ (by increasing the transmit power over relay hops and changing the constellation size and code rate accordingly) or decrease $\kappa_{1}, \kappa_{2}$ at the expense of decreased $\eta$ (by decreasing the transmit power over the relay hops and adjusting constellation and code rate accordingly). That is the tradeoff that has to be made.

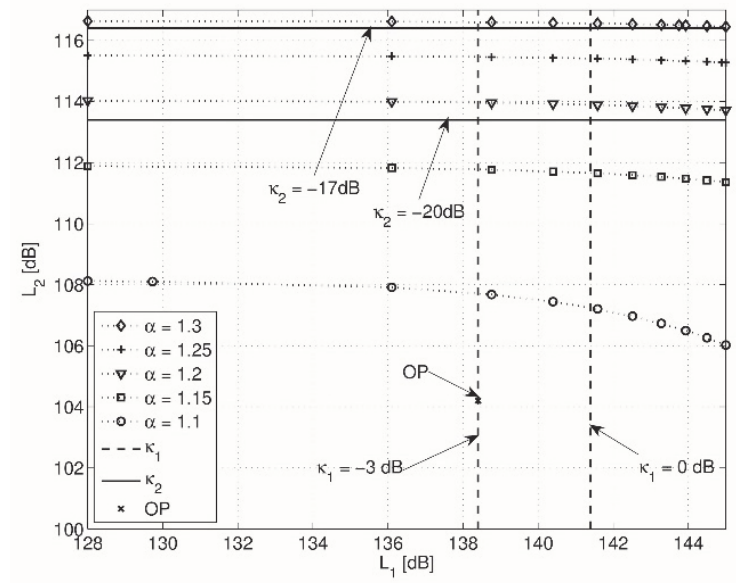

Fig. 7. $\left(L_{1}, L_{2}\right)$ space, $\tau=-3 \mathrm{~dB}$ : $\alpha$-levels overlapped with $\kappa_{1}, \kappa_{2}$ lines.

\section{DISCUSSION}

The trade-off between EE and generated interference at an unintended receiver $\mathrm{A}$, using RR, depends on a number of conditions and parameters. If only LOS/NLOS conditions along each of the 5 relevant links (BS-UE, BS$\mathrm{RN}, \mathrm{RN}-\mathrm{UE}, \mathrm{BS}-\mathrm{A}$ and RN-A) are considered, there are $2^{5}=32$ possibilities. For each link, the probability of a certain LOS/NLOS condition depends on the sourcedestination separating distance [9]. We emphasize the most probable outcomes.

Within the RN pico-cell, e.g. beyond $60 \%$ of the radius of the cell of interest $(300 \mathrm{~m}$ for radius of $500 \mathrm{~m})$, the BSUE link is NLOS with high probability [9]. Beyond the cell edge, the BS-A link is NLOS with high probability.

Then, the worst case for EE increase is NLOS/NLOS along both BS-RN and RN-UE. The BS-RN link is subject to network design, so the probability of LOS is not negligible. In case of NLOS BS-RN link, the shadowing effect may be small with non-negligible probability. The RN-UE link also has non-negligible probability of either LOS or NLOS with a small shadowing effect.

The RN-A link beyond the cell edge is NLOS with high probability (case $\mathrm{c} 4$ ), but there may be areas with either LOS or NLOS with low shadowing (case c3). The case c4 is amenable to the EE-SE vs generated interference tradeoff because of the large channel loss $L_{\mathrm{A} 2}$, which may yield a sufficiently large $\tau$. However, the case c 3 is not, because of the large generated interference from the RN, i.e. small $\tau$.

If RR increases EE, it is due to: $L_{1}<L, \Delta g^{\mathrm{dB}}=g_{\mathrm{R}}{ }^{\mathrm{dB}}-g_{\mathrm{D}} \mathrm{dB}$ $>0 \mathrm{~dB}$ and adjustment of the constellation size and code rate over both relay hops [4].

However, doubling of the data rate ratio $\beta$ requires, on average, a $6 \mathrm{~dB}$ increase in transmit power, whereas the same increase in transmit power quadruples the generated interference. Therefore, it is essential to place the $\mathrm{RN}$ in a cell such that the channel losses along BS-RN and RN-UE links (within the $\mathrm{RN}$ pico-cell) are minimized, whereas channel losses from the $\mathrm{RN}$ to unintended receivers (out of the RN pico-cell) are as large as possible. If this is not possible, then the use of a small BS (with wireline backhaul) instead of a $\mathrm{RN}$ is recommended.

\section{CONCLUSION}

When using the RR, a trade-off between increased EE vs the reduced generated interference is possible. It has been quantified and key parameters have been assessed in this paper. The key to achieving a good trade-off is appropriate placement of the $\mathrm{RN}$ and exploitation of the differences in channel losses along different links: low losses for desirable links and high losses for undesirable links. A complete assessment requires integration over the areas of interest, which is a goal of the future work.

\section{ACKNOWLEDGMENT}

Discussed at the IC1004 COST Action in SWG 2.1 PHY layer Cooperation and Relaying.

\section{REFERENCES}

[1] Y. Yuan, LTE-Advanced Relay Technology and Standardization. Springer, 2013.

[2] C. Hoymann, W. Chen, J. Montojo, A. Golitschek, C. Koutsimanis, and X. Shen, "Relaying operation in 3GPP LTE: challenges and solutions," IEEE Comm. Mag., vol. 50, no. 2, Feb. 2012, pp. 156162.

[3] L. M. Correia, D. Zeller, B. Oliver, D. Ferling, Y. Jading, I. Gódor, G. Auer, and L. Van Der Perre, "Challenges and enabling technologies for energy aware mobile radio networks," IEEE Comm. Mag., vol. 48, no. 10, Nov. 2010, pp. 66-72.

[4] G. Dimić, D. Bajić, and M. Beko, "Relay Type 1a in LTEAdvanced: Can It Increase Energy Efficiency?" 31st URSI GASS, Radio-Communication Systems and Signal Processing: Cooperative Communications and Network Coding, Beijing, China, Aug 16-23, 2014, C05:3.

[5] A. Lozano, R. W. Heath, and J. G. Andrews, "Fundamental Limits of Cooperation," IEEE Trans. on Info. Theory, vol. 59, no. 9, Sep. 2013, pp. 5213-5226.

[6] M. Dohler and Y. Li, Cooperative Communications: Hardware, Channel and PHY. Wiley, 2010.

[7] M. A. Imran, E. Katranas and (Eds.), "Energy efficiency analysis of the reference systems, areas of improvements and target breakdown," EARTH FP7 Project, Deliverable D2.3 v2, Brussels, Belgium, https://www.ict-earth.eu, Jan. 2012.

[8] G. Dimić, N. Zogović, and D. Bajić, "Supportive relay with heterogeneous transceivers: quantification of energy efficiency improvement," IEEE ICC 2013 - E2Nets Workshop, Budapest, Hungary, June 9, 2013.

[9] Z. Wang, E. K. Tameh, and A. R. Nix, "Statistical peer-to-peer channel models for outdoor urban environments at $2 \mathrm{GHz}$ and 5GHz," Vehicular Tech. Conf. - Fall 2004, Los Angeles, CA, USA, 26-29 September 2004.

[10] ETSI, "LTE; Evolved universal terrestrial radio access (E-UTRA); base station (BS) radio transmission and reception (3GPP TS 36.104 version 9.4.0 Release 9)," Sophia Antipolis Cedex - France, 2010-07. 